\title{
Incidence of High Altitude Illnesses among Unacclimatized Persons Who Acutely Ascended to Tibet
}

\author{
Yusheng Ren, ${ }^{1}$ Zhongming Fu, ${ }^{2,3}$ Weimin Shen, ${ }^{4}$ Ping Jiang, ${ }^{3}$ \\ Yanlin $\mathrm{He}^{2}{ }^{2}$ Shaojun Peng, ${ }^{2}$ Zonggui Wu, ${ }^{1}$ Bo $\mathrm{Cui}^{5}$
}

\begin{abstract}
Ren, Yusheng, Zhongming Fu, Weimin Shen, Ping Jiang, Yanlin He, Shaojun Peng, Zonggui Wu, and Bo Cui. Incidence of high altitude illnesses among unacclimatized persons who acutely ascended to Tibet. High Alt. Med. Biol. 11:39-42, 2010.- High altitude illnesses pose health threats to unwary travelers after their acute ascent to high altitude locations. The incidence of high altitude illnesses among unacclimatized persons who acutely ascend to Tibet has not been previously reported. In the present study, we surveyed the incidence of high altitude illness among 3628 unacclimatized persons who had no previous high altitude experience and who traveled to Tibet by air to an altitude of $3600 \mathrm{~m}$. These subjects were asked to answer questions in a written questionnaire about symptoms associated with high altitude illnesses that occurred within 2 weeks of their first arrival, their severity, and possible contributing factors. Physical examination and appropriate laboratory tests were also performed for hospitalized subjects. We found that 2063 respondents had mild acute mountain sickness with an incidence of $57.2 \%$, and $249(12.07 \%)$ of them were hospitalized for treatment. The incidence of high altitude pulmonary edema was $1.9 \%$, while no case of high altitude cerebral edema was found. Additionally, there was no report of death. Psychological stresses and excessive physical exertions possibly contributed to the onset of HAPE. Acute mountain sickness is common among unacclimatized persons after their acute ascent to Tibet. The incidence of HAPE and HACE, however, is very low among them.
\end{abstract}

Key Words: incidence; acute mountain illnesses; Tibet

\section{Introduction}

$\mathbf{W}$ ITH THE INCREASING POPULARITY OF OUTDOOR excursions and easier access to high altitude regions for low altitude inhabitants, there are millions of visitors to high altitude locations each year. However, maladaptation to rarefied high altitude environments among unacclimatized persons can lead to a multitude of health problems, including acute mountain sickness (AMS), high altitude cerebral edema (HACE), and high altitude pulmonary edema (HAPE). These problems are of public concern as they have economic implications for the ski industry and the travel industry in the high altitude regions of the world. Additionally, HAPE and HACE, though uncommon, pose a severe health threat to unwary travelers as they can be rapidly fatal.
AMS is characterized by the presence of headache in an unacclimatized person who also has one or more of the following symptoms: nausea, anorexia, vomiting, insomnia, or dizziness (Hackett and Roach, 2001). When ataxia, hallucinations, altered consciousness, or other neurologic signs develop in an unacclimatized person with AMS or HAPE, HACE is diagnosed until proved otherwise (Hackett and Roach, 2004). HAPE is suspected when exertional dyspnea, moist cough, or other symptoms are found that suggest the presence of pulmonary edema (Maggiorini, 2006). The incidence of AMS correlates with altitude (Hackett et al., 1976). AMS occurred in 9\% of climbers in the Alps at an altitude of $2850 \mathrm{~m}$, which increased to $13 \%$ and $34 \%$ at an altitude of 3050 and $3650 \mathrm{~m}$, respectively (Maggiorini et al., 1990). HAPE and HACE are less frequent than AMS. HAPE and HACE

\footnotetext{
${ }^{1}$ Department of Cardiology, Shanghai Changzheng Hospital, Second Military Medical University, Shanghai, China.

${ }^{2}$ Department of Medicine, 8th Hospital of PLA, Rikaze, Tibet, China.

${ }^{3}$ Department of Medicine, 41st Hospital of PLA, Shannan, Tibet, China.

${ }^{4}$ Department of Anesthesiology, 41st Hospital of PLA, Qingdao, Shandong, China.

${ }^{5}$ Departments of Surgery and Pathology, Duke University Medical Center, Durham, North Carolina, USA.
} 
occurred in $7(2.52 \%)$ and $5(1.80 \%)$ of 278 unacclimatized hikers at an altitude of $4243 \mathrm{~m}$ (Hackett et al., 1976).

Tibet is increasingly becoming a tourist destination for many domestic and international travelers. However, there has been no previous report of the incidence of high altitude illnesses among unacclimatized travelers who inhabit lowaltitude regions and who travel by air to Tibet. Here we report our results from a health survey of 3628 unacclimatized subjects on symptoms associated with high altitude illnesses and their possible contributing factors. We find that mild AMS is very common among unacclimatized subjects with no previous high altitude experience, whereas the incidence of HAPE and HACE is extremely low among these subjects. Additionally, HAPE is associated with psychological stresses, fatigue, excessive physical activities, and lack of rest before traveling to high altitude regions. To our knowledge, this is by far the largest survey of high altitude illnesses among unacclimatized persons who acutely ascended to high altitude anywhere in the world. Our results provide valuable information on the prospective management of unacclimatized travelers contemplating acute ascent to Tibet.

\section{Methods}

\section{Subjects}

New army recruits $(n=3628)$ who had no previous exposure to high altitude environments were included in the current study. These participants inhabited low-altitude regions with an altitude of 100 to $300 \mathrm{~m}$ above sea level, and they underwent acute ascent to altitudes above $3600 \mathrm{~m}$ by air transportation. A total of 3628 questionnaires with questions about symptoms associated with high altitude illnesses (Hackett and Roach, 2001) that occurred within 2 weeks of their first arrival, their severity, and possible contributing factors were distributed to these subjects and recollected, and $99.36 \%$ (3605) of the returned questionnaires were qualified for further statistical analysis. The psychological stresses were measured by self-report of the presence or absence of psychological stresses in the participants after their ascent to high altitude. The respondents consisted of 3594 males, age ranging from 16 to $22 \mathrm{yr}$ and with an average age of $18.6 \mathrm{yr}$, and 9 females, age ranging from 17 to $20 \mathrm{yr}$ and with an average age of 18.2 yr. There were 3548 Han Chinese respondents and 67 respondents of other ethnic minorities. AMS, HAPE, and HACE were diagnosed according to the Criteria for the Denomination, Classification and Diagnosis of High Altitude Illnesses set forth by the 1996 Ad Hoc Committee on High Altitude Illnesses of Chinese Medical Association (Tables 1 and 2) (Third Ad Hoc Committee on High Altitude Illnesses of Chinese Medical Association, 1996). Specifically, mild AMS was diagnosed as the presence of headache or vomiting that affected the functioning of daily activities. When either symptom was absent, a score between 5 and 10 was required for the diagnosis of mild AMS, with one score assigned for each of the following symptoms: fatigue, nausea, palpitation, shortness of breath, chest tightness, dizziness, loss of appetite, bloating, diarrhea, constipation, bluish coloration of lips, drowsiness, and numbness or tingling in the hands or feet. HAPE was diagnosed when dyspnea at rest, moist cough or frothy pink sputum, central cyanosis, or rales were present and confirmed by $x$-ray examinations. Chest $X$ rays were read by two experienced radiologists who were blind to the patient status. The presence of myocardial infarction, cardiac failure, or other cardiopulmonary diseases was excluded by physical examination, electrocardiographic (ECG), and other appropriate laboratory tests. HACE was diagnosed when a patient with AMS or HAPE developed neurologic signs, such as ataxia or altered consciousness. The presence of stroke, carbon monoxide poisoning, seizures, meningitis, and encephalitis was excluded.

Written informed consent for participating in the survey was obtained from all study participants in accordance with the guidelines set by the hospital ethics review committee. The current study protocol was approved by the institution review board.

\section{Statistical analysis}

Data were entered into EPI Info 5.0 database and analyzed using SAS 6.12. Data were expressed in mean \pm standard deviation, and differences between groups were analyzed using a $\chi$-square test.

Table 1. Acute Mountain Illness Scoring System

\begin{tabular}{lc}
\hline Symptoms & Severity \\
\hline Headache & Score \\
Not noticeable and no suffering. Does not interfere with daily activities. & + \\
Mild and suffering. Headache is relieved after pain medication & + \\
$\quad$ and does not interfere with daily activities. & + \\
Moderate and suffering. Headache is relieved after pain medication & +++ \\
$\quad$ and does not interfere with daily activities. & + \\
$\quad$ Severe and incapacitating. No relief after pain medication. & ++ \\
Vomiting & +++ \\
Vomiting 1 to 2 times/day, which does not interfere with daily activities. & 7 \\
Vomiting 3 to 4 times/day, which interferes with daily activities. & 2 \\
Vomiting more than 3 times/day; patients confined to bed & 4 \\
$\quad$ and no relief after medication. & 7 \\
Others & + \\
One score for each of the following symptoms: dizziness, nausea, palpitation, \\
$\quad$ shortness of breath, chest tightness, poor appetite, diarrhea, constipation, cyanosis, \\
$\quad$ and numbness of the hand or foot.
\end{tabular}


Table 2. Severity of Acute Mountain Sickenss

\begin{tabular}{|c|c|}
\hline Severity & Scoring \\
\hline Normal $( \pm)$ & 1 to 4 \\
\hline Mild (+) & $\begin{array}{l}\text { Headache }(+) \text { or vomiting }(+) \\
\quad \text { or total score } 5 \text { to10 }\end{array}$ \\
\hline Moderate $(++)$ & $\begin{array}{l}\text { Headache }(++) \text { or vomiting }(++) \\
\quad \text { or total score } 11 \text { to } 15\end{array}$ \\
\hline Severe $(+++)$ & $\begin{array}{l}\text { Headache }(+++) \text { or vomiting }(+++) \\
\quad \text { or total score }>16\end{array}$ \\
\hline
\end{tabular}

The authors had full access to and took full responsibility for the integrity of the data. All authors have read and agreed to the manuscript as written.

\section{Results}

Our survey has identified bluish coloration of the lips in the unacclimatized subjects as the most frequent symptom, which was reported by $95.31 \%$ of the respondents, reflecting the hypoxic environment in Tibet. Headache was the second most frequent symptom experienced by the unacclimatized subjects, with $74.98 \%$ of those surveyed having at least one episode of headache. Further, severe or incapacitating headaches were found in $6.99 \%$ of those surveyed (Table 3). The third most frequent symptom reported by the respondents was loss of appetite, which was seen in $67.74 \%$ of the respondents. In addition, palpitation and chest tightness were observed in slightly more than half of those surveyed. Mild AMS was diagnosed in 2063 (57.2\%) cases, and 249 (12.07\%) of them were hospitalized. The rest of the cases only received symptomatic management. Seven $(1.9 \%)$ cases of HAPE were diagnosed. The hospital stay for patients with mild AMS was $3.2 \pm 1.2$ days and that for patients with HAPE was $4.6 \pm 2.1$ days. No case of HACE was found in the respondents. Additionally, there was no report of death among the respondents.

We also investigated possible contributing factors to the onset of AMS and HAPE (Table 4). We found that lack of rest before ascent to high altitude regions was present in $100 \%$ of patients with HAPE and $66.5 \%$ of those with mild AMS and $5.86 \%$ of those free of HAPE or AMS. In addition, psychological stresses were identified in a greater portion of patients with HAPE $(71.42 \%)$ than those with mild AMS (4.31\%). Fatigue

TABle 3. Symptoms EXPERIENCEd by UNACCLIMATIZED Subjects in Tibet $(\mathrm{N}=3605)$

\begin{tabular}{lrrr}
\hline & \multicolumn{3}{c}{ No. of cases (\%) } \\
\cline { 2 - 4 } Symptoms & \multicolumn{1}{c}{ Mild } & Moderate & Severe \\
\hline Headache & $1694(47.0)$ & $757(20.99)$ & $252(7.0)$ \\
Nausea & $757(21.0)$ & $180(4.99)$ & $36(1.0)$ \\
Vomiting & $397(11.0)$ & $36(0.99)$ & $0(0)$ \\
Palpitations & $1730(48.0)$ & $396(11.00)$ & $11(0.3)$ \\
Shortness of breath & $577(16.01)$ & $324(9.0)$ & $37(1.0)$ \\
Chest tightness & $1406(39.0)$ & $433(12.0)$ & $22(0.6)$ \\
Poor appetite & $2028(56.26)$ & $649(18.0)$ & $396(11.0)$ \\
Bluish lips & $3281(91.0)$ & $151(4.2)$ & $4(0.4)$ \\
Dyspnea & $937(26.0)$ & $188(5.2)$ & $18(0.5)$ \\
Others & $725(20.1)$ & $40(1.1)$ & $3(0.1)$ \\
\hline
\end{tabular}

Table 4. Possible Contributing Factors in High Altitude Illnesses ( $\mathrm{N}=3605)$

\begin{tabular}{lrc}
\hline & \multicolumn{2}{c}{ No. of cases (\%) } \\
\cline { 2 - 3 } Risk factors & Mild AMS & HAPE \\
\hline Fatigue & $121(5.86)$ & $4(57.14)$ \\
Smoking & $96(4.65)$ & $2(28.57)$ \\
Drinking & $12(0.58)$ & $1(14.28)$ \\
Psychological stresses & $89(4.31)$ & $5(71.42)$ \\
Lack of rest before trip & $1372(66.50)$ & $7(100.0)$ \\
Excessive activities at high altitude & $84(4.07)$ & $4(57.14)$ \\
\hline
\end{tabular}

and excessive physical activities after arrival in high altitude regions were observed in more than half of those with HAPE, but were only seen in less than $6 \%$ of those with mild AMS.

\section{Discussion}

AMS is a self-limiting disease. Prevention involves natural acclimatization by gradual ascent to high altitude or a short course of intermittent hypoxia training before the trip. In addition, management of AMS consists of avoidance of further ascent to a higher altitude. If AMS fails to resolve with symptomatic treatment, descent to a lower altitude is advised. The results of our clinical investigation indicate that AMS is very common among unacclimatized persons, with close to $80 \%$ of those surveyed diagnosed with mild or moderate AMS. The incidence is markedly higher than that reported for unacclimatized travelers $(34 \%)$ at a similar altitude (Maggiorini et al., 1990). This notable difference can be attributed to the different scoring systems for diagnosing AMS. The Lake Louise scoring system is generally used internationally (Maggiorini et al., 1998; Dellasanta et al., 2007), which requires the presence of headache and at least one other symptom, such as nausea or vomiting, with the severity rated on a scale of 1 to 3 (Bartsch et al., 1989). We have employed a generally accepted AMS scoring system set forth by the 1996 Ad Hoc Committee on High Altitude Illnesses of Chinese Medical Association (Third Ad Hoc Committee on High Altitude Illnesses of Chinese Medical Association, 1996), which requires the presence of headache or vomiting that incapacitates a patient for diagnosing AMS. In the absence of headache or vomiting, the presence of at least five other symptoms, such as nausea, fatigue, or loss of appetite, is required for the diagnosis of mild AMS. This Chinese scoring system is more inclusive than the Lake Louise scoring system. Further, the Chinese scoring system is less subjective as it does not involve scoring the severity of symptoms, whereas the Lake Louise scoring system is based on the quantification of the reported subjective sensation of the severity of symptoms. On the other hand, the incidence of HAPE among our respondents is markedly lower than that reported by others (Hackett et al., 1976), and we have observed no case of HACE. This may be related to the relative young age of our respondents, who have an average age of only $18.6 \mathrm{yr}$, and the emphasis by our medical institutions on early recognition of HACE and active management of moderate and severe AMS. We further found that lack of adequate rest before ascent to high altitude regions may be a contributing factor in both mild AMS and HAPE. On the other hand, psychological stresses and physical exertions also appear to contribute to HAPE. 
High altitude illnesses put an economic burden on the tourist industry in high altitude regions and pose a health threat to the unwary traveler to high altitude locations. Our findings reveal that AMS is more common than previously thought. Physical adaptation by gradual ascent to a higher altitude is generally recommended to avoid the onset of AMS, HAPE, and HACE. Our findings also suggest that minimization of physical activities is advisable for unacclimatized persons after their arrival at high altitude locations, especially within the first $48 \mathrm{~h}$ of their arrival. A greater proportion of patients with HAPE experienced psychological stresses during their ascent to high altitude regions. Psychological adaptation by persons with no previous high altitude experience may be needed just as for physical adaptation.

In summary, we report the results of our survey of high altitude illnesses in the largest cohort of unacclimatized persons in high altitude regions and provide further insight that AMS is commonly seen among unacclimatized persons after their acute ascent to high altitude regions. Minimization of possible factors associated with or contributing to the onset of AMS and HAPE and physical adaptation and psychological adaptation are recommended to lessen the occurrence of high altitude illnesses. With the increasing popularity of Tibet as a tourist destination, our report provides much needed information about the incidence of high altitude illnesses among unacclimatized travelers to Tibet and possible contributing factors to AMS and HAPE.

\section{Disclosures}

The authors have no conflicts of interest or financial ties to disclose.

\section{References}

Bartsch P., Lammle B., Huber I., Haeberli A., Vock P., Oelz O., and Straub P.W. (1989). Contact phase of blood coagulation is not activated in edema of high altitude. J. Appl. Physiol. 67:1336-1340.

Dellasanta P., Gaillard S., Loutan L., and Kayser B. (2007). Comparing questionnaires for the assessment of acute mountain sickness. High Alt. Med. Biol. 8:184-191.

Hackett P.H., Rennie D., and Levine H.D. (1976). The incidence, importance, and prophylaxis of acute mountain sickness. Lancet. 2:1149-1155.

Hackett P.H., and Roach R.C. (2001). High-altitude illness. N. Engl. J. Med. 345:107-114.

Hackett P.H., and Roach R.C. (2004). High altitude cerebral edema. High Alt. Med. Biol. 5:136-146.

Maggiorini M. (2006). High altitude-induced pulmonary oedema. Cardiovasc. Res. 72:41-50.

Maggiorini M., Buhler B., Walter M., and Oelz O. (1990). Prevalence of acute mountain sickness in the Swiss Alps. BMJ 301:853-855.

Maggiorini M., Muller A., Hofstetter D., Bartsch P., and Oelz O. (1998) Assessment of acute mountain sickness by different score protocols in the Swiss Alps. Aviat. Space Environ. Med. 69:1186-1192.

Third Ad Hoc Committee on High Altitude Illnesses of Chinese Medical Association. (1996). Consensus statement for the criteria for the denomination, classification and diagnosis of high altitude illnesses in China. Tibetan Med. J. 17:1-3.

Address correspondence to: Dr. Yusheng Ren Department of Cardiology, Shanghai Changzheng Hospital Second Military Medical University 415 Fengyang Rd., Shanghai, 200003, China

E-mail: renyusheng@gmail.com

Received July 20, 2009; accepted in final form December 7, 2009. 
This article has been cited by:

1. C. M. Beall, G. L. Cavalleri, L. Deng, R. C. Elston, Y. Gao, J. Knight, C. Li, J. C. Li, Y. Liang, M. McCormack, H. E. Montgomery, H. Pan, P. A. Robbins, K. V. Shianna, S. C. Tam, N. Tsering, K. R. Veeramah, W. Wang, P. Wangdui, M. E. Weale, Y. Xu, Z. Xu, L. Yang, M. J. Zaman, C. Zeng, L. Zhang, X. Zhang, P. Zhaxi, Y. T. Zheng. 2010. Natural selection on EPAS1 (HIF2 ) associated with low hemoglobin concentration in Tibetan highlanders. Proceedings of the National Academy of Sciences 107:25, 11459-11464. [CrossRef] 